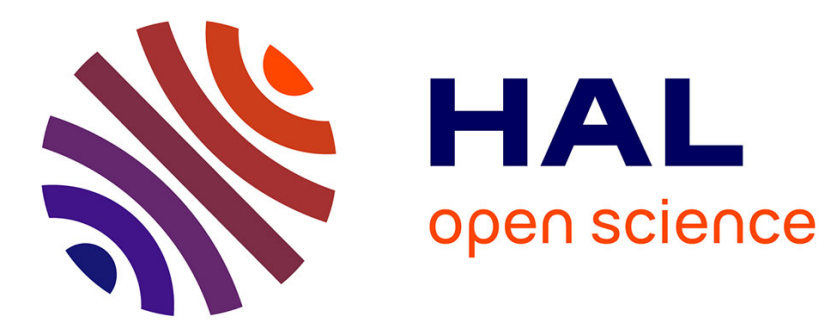

\title{
Le transcodage de l'axe II : une avancée utile
}

\author{
Y. Coinçon, B. Durand, R. Misès, M. Botbol, C. Bursztejn, J. Garrabé, N. \\ Garret Gloanec, B. Golse, C.H. Portelli, J.-P. Raynaud, et al.
}

\section{To cite this version:}

Y. Coinçon, B. Durand, R. Misès, M. Botbol, C. Bursztejn, et al.. Le transcodage de l'axe II: une avancée utile. Annales Médico-Psychologiques, Revue Psychiatrique, 2011, 10.1016/j.amp.2011.03.010 . hal-00748144

\section{HAL Id: hal-00748144 \\ https://hal.science/hal-00748144}

Submitted on 5 Nov 2012

HAL is a multi-disciplinary open access archive for the deposit and dissemination of scientific research documents, whether they are published or not. The documents may come from teaching and research institutions in France or abroad, or from public or private research centers.
L'archive ouverte pluridisciplinaire HAL, est destinée au dépôt et à la diffusion de documents scientifiques de niveau recherche, publiés ou non, émanant des établissements d'enseignement et de recherche français ou étrangers, des laboratoires publics ou privés. 


\section{Accepted Manuscript}

Title: Le transcodage de l'axe II : une avancée utile

Authors: Y. Coinçon, B. Durand, R. Misès, M. Botbol, C. Bursztejn, J. Garrabé, N. Garret Gloanec, B. Golse, C.H. Portelli, J.-P. Raynaud, G. Schmit, J.-P. Thévenot

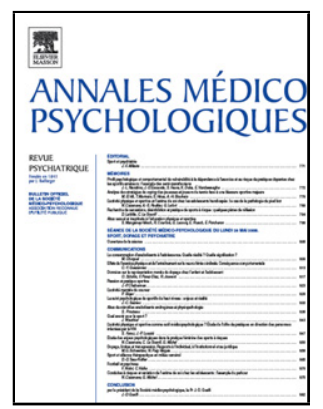

PII: $\quad$ S0003-4487(11)00075-8

DOI: $\quad$ doi:10.1016/j.amp.2011.03.010

Reference: $\quad$ AMEPSY 1316

To appear in: $\quad$ Annales Médico-Psychologiques

Please cite this article as: Coinçon Y, Durand B, Misès R, Botbol M, Bursztejn C, Garrabé J, Garret Gloanec N, Golse B, Portelli CH, Raynaud J-P, Schmit G, Thévenot J-P, Le transcodage de l'axe II : une avancée utile, Annales medio-psychologiques (2010), doi:10.1016/j.amp.2011.03.010

This is a PDF file of an unedited manuscript that has been accepted for publication. As a service to our customers we are providing this early version of the manuscript. The manuscript will undergo copyediting, typesetting, and review of the resulting proof before it is published in its final form. Please note that during the production process errors may be discovered which could affect the content, and all legal disclaimers that apply to the journal pertain. 
Communication

Le transcodage de l'axe II : une avancée utile

Axis II transcoding: A useful initiative

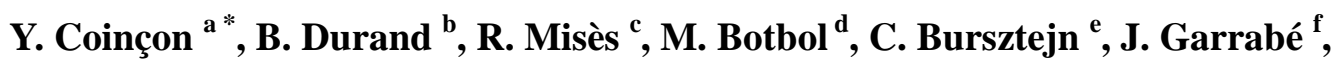
N. Garret Gloanec ${ }^{\text {g }}$, B. Golse ${ }^{\text {h }}$, Ch. Portelli ${ }^{\text {i }}$, J.-P. Raynaud ${ }^{\text {j, G. Schmit }}{ }^{\text {k, J.- }}$ P. Thévenot ${ }^{1}$

a) Dr Yvonne Coinçon, pédopsychiatre, chef de secteur de psychiatrie infanto-juvénile, 8 rue Servan 38000 Grenoble, France

b) Dr B. Durand, 121 avenue d’Italie, 75013 Paris, France

c) Pr Roger Misès, 21 rue Barbet-de-Jouy, 75007, Paris, France

d) Dr Michel Botbol, Psychiatre des Hôpitaux, Clinique Dupré, 30 avenue F.-Roosevelt, 92331 Sceaux cedex, France

e) Pr Claude Bursztejn, CHRU Hôpital civil, 1 place de l'Hôpital, 67091 Strasbourg cedex, France

f) Dr Jean Garrabé, Psychiatre des Hôpitaux, 7, place Pinel, 75013 Paris, France

g) Dr Nicole Garret Gloanec, P.H., Centre nantais de la parentalité, 27, square La Pérouse, 44000 Nantes, France

h) Dr Bernard Golse, pédopsychiatre, hôpital Necker, 149 rue de Sèvres, 75743 Pais cedex 15, France

i) Dr Christian Portelli, Psychiatre des Hôpitaux, Médecin Directeur du CMPP de Boulogne, 12 rue Émile Landrin, 92100 Boulogne-Billancourt, France

j) Dr Jean-Philippe Raynaud, CHU de Toulouse Hôpital La Grave, TSA 60033, 31059 Toulouse cedex 9, France

k) Dr Gérard Schmit, CHRU Hôpital R. Debré, avenue du Général Koenig, 51092 Reims cedex, France

l) Dr Jean-Pierre Thévenot, Psychiatre des Hôpitaux, CMP, 20 villa Compoint, 75017 Paris, France

Auteur correspondant : Dr Yvonne Coinçon, chef de secteur de psychiatrie infanto-juvénile, 8 rue Servan 38000 Grenoble, France

Adresse email : $\underline{\text { Yvonne-coincon@wanadoo.fr }}$ 


\title{
Résumé
}

La première table de correspondance établie en 2007 entre la CFTMEA et la CIM-10 était loin d'être exhaustive et ne concernait que l'Axe I. La Révision 2010 de la CFTMEA, qui permet un transcodage terme à terme, rend les deux classifications compatibles dans la pratique quotidienne et propose également un transcodage à l'Axe II.

Les facteurs associés, à la fois somatiques et d'environnement répertoriés dans l'Axe II de la CFTMEA, sont disséminés dans les 21 chapitres de la CIM-10, ce qui rend leur repérage difficile en pratique courante. La simplicité d'utilisation de l'Axe II permet d'éviter que ces facteurs soient ignorés ou négligés par les cliniciens et facilite la tâche des chercheurs.

M ots clés : Adolescent ; CIM-10 ; Classification ; Enfant ; Transcodage

\begin{abstract}
The first corresponding table between CFTMEA and ICD-10 was established in 2007. But it was far from exhaustive and only concerned Axis I. The 2010 CFTMEA revision enables item-to-item transcoding and makes the two classifications compatible in daily practice. It also permits Axis II transcoding.

Both somatic and environment associated factors, as listed in CFTMEA's Axis II, are scattered throughout the twenty one ICD-10 chapters, which makes their identification difficult in practice. The simplicity of using Axis II avoids these factors being ignored or neglected by clinicians and makes it easier for researchers.
\end{abstract}

Keywords: Adolescent; Child; Classification; ICD-10; Transcoding

La révision 2000 de la Classification française des troubles mentaux de l'enfant et de l'adolescent (CFTMEA) n'avait pas proposé de correspondance avec la CIM-10. Néanmoins, les contraintes imposées par l'administration sanitaire avec la mise en œuvre du RIM.PSY en 2006-2007 qui exigeaient que les praticiens recourent exclusivement à la CIM-10 ont nécessité de rapprocher la CFTMEA de la classification internationale et d'établir des correspondances entre elles. Cela était d’autant plus nécessaire que la CIM-10 ne répondait pas aux exigences cliniques de la pédopsychiatrie et de sa psychopathologie.

Dans un premier temps, en 2007, une table de correspondance entre la CFTMEA et la CIM-10 a été établie et publiée sur le site de l'Agence Technique de l’Information 
Hospitalière (ATIH), mais, d'une part, cette correspondance était loin d'être exhaustive, et, d'autre part, elle ne concernait que l'Axe I.

En effet, restreint à l’Axe I, le tableau de transcodage ne répond qu’à la seule lecture sémiologique de la clinique et ne permet pas d'appuyer cette classification sur un modèle pluridimensionnel des pathologies, de leurs prises en charge et de leurs évolutions, en recueillant les informations sur les facteurs associés, médicaux et environnementaux comme le permet l'Axe II de la CFTMEA.

Actuellement, en effet, d'aucuns, sous la pression des pouvoirs publics et d'un renoncement implicite à la psychopathologie, se réfèrent à la CIM-10. Faut-il encore qu'ils utilisent correctement cette classification, car cela suppose de la connaître et d'utiliser les milliers de pages des trois manuels qui la composent: une table analytique, un manuel d'utilisation et un index alphabétique.

Cela est évident lorsque l'on veut collecter les facteurs associés, éventuellement étiologiques, qu'ils soient somatiques ou d'environnement qui correspondent à l'Axe II de la CFTMEA. Relever les facteurs somatiques, que ce soit dans le cadre du recueil d'activités des services ou pour des travaux de recherche, impose de se référer aux 21 chapitres de la CIM10, dont le chapitre 20 consacré aux causes externes de morbidité et de mortalité. Quant aux facteurs d'environnement, dont on sait l'importance qu'ils revêtent en psychopathologie de l'enfant, il faut recourir au chapitre 21 de la CIM consacré aux « Facteurs influant sur l'état de santé et autres motifs de recours aux services de santé », coté en Z (de Z00 à Z99).

Dans la pratique quotidienne, ce travail n'est guère possible, d'autant que les équipes de psychiatrie infantojuvénile ne disposent le plus souvent que du seul chapitre 7 consacré à la psychiatrie, et n’ont pas la possibilité immédiate, à supposer qu'elles le souhaitent, de consulter les autres volumes de la CIM-10. D’où le risque que ces facteurs associés ne soient pas répertoriés.

Or, les items de l'Axe II de la CFTMEA regroupent l'ensemble des données disséminées dans la CIM-10, ce qui facilite leur repérage sur le plan nosographique (tableau 1). S’il ne s'agit pas de faire correspondre les facteurs identifiés à telle ou telle pathologie précise, comme le confirment d'ailleurs les avancées de la recherche dans le domaine des neurosciences et de la génétique qui ont connu un essor remarquable au cours des trente dernières années, ceux-ci constituent des facteurs de vulnérabilité importants à repérer dans une démarche de prévention et d'éducation thérapeutique. La correspondance qui est proposée avec cette table de transcodage de l'Axe II devrait réduire le risque que le repérage de ces 
facteurs associés soit ignoré ou négligé du fait de la simplicité d’utilisation de l’Axe II de la CFTMEA.

Les items de l'Axe II de la CFTMEA permettent, grâce aux études statistiques, de se dégager d’hypothèses causalistes. Ils montrent qu'un même facteur somatique peut être relevé chez des patients aux profils psychopathologiques différents : il en est ainsi, par exemple, pour la prématurité ou les infections ORL précoces à répétition. De même en ce qui concerne les facteurs d'environnement : un deuil peut être retrouvé dans les antécédents de vie au cours d’un épisode dépressif aussi bien que dans la mise en place d'une angoisse de séparation chez un enfant né postérieurement au décès en question, ou encore des dysfonctionnements relationnels précoces sont retrouvés dans la biographie de certains patients porteurs d'un déficit global du développement, et d'autres d'une dysharmonie psychotique (également appelée, dans la révision 2010, dysharmonie multiple et complexe du développement).

Cette avancée dans la correspondance entre les deux classifications devrait faciliter le repérage de ces facteurs associés par la simplicité d’utilisation de cet Axe II de la CFTMEA, tant dans la pratique quotidienne que dans les travaux de recherches cliniques et épidémiologiques, tout en permettant de se conformer aux exigences de l'administration.

À la différence de l'Axe I où les catégories un à quatre s'excluent mutuellement, et les catégories cinq à neuf ne sont retenues que si aucune des précédentes catégories, structurales, ne l'a été, celles de l'Axe II ne s'excluent pas. Elles peuvent se cumuler entre elles, sans limite de nature et de quantité.

Compte tenu de cette caractéristique, il est recommandé de ne retenir, en pratique clinique, que les facteurs dont l'incidence sur la genèse ou l'évolution des troubles est considérée comme possible ou certaine. En revanche, dans les travaux épidémiologiques, le cumul de tous les items de l'Axe II pour tous les cas inclus garde sa validité et prend toute sa valeur pour affiner les connaissances sur ces pathologies et leur contexte de développement.

Pour conclure cette présentation, rappelons que les critères retenus en matière de classification peuvent être en rapport avec le type de pathologie (tumeurs néoplasiques), l’organe concerné (pathologies cardiaques), ou encore une catégorie de patients (pédiatrie, gérontologie). La classification peut alors non seulement traduire le diagnostic principal, mais indiquer aussi qu'un diagnostic différentiel a été envisagé.

Mais lorsqu'une classification renonce à choisir des critères de référence et se réclame même d'une position « athéorique », comme le DSM, avec l'objectif principal de simplement identifier et lister des signes avec une fiabilité interjuge satisfaisante, cela rend nécessaire de 
repérer ce qu’il est convenu d'appeler des «pathologies associées » de plus en plus nombreuses pour chaque patient.

L’objectif poursuivi en proposant pour la première fois un transcodage pour les Axes I et II est donc de faciliter la tâche des praticiens, tout en réservant la possibilité de mener des travaux de recherche clinique et/ou épidémiologique sur la base d'un instrument unique, forgé par des utilisateurs aux prises avec les réalités de la pratique soignante et de la recherche.

Nous attendons des utilisateurs qu'ils nous transmettent leurs observations afin que les améliorations qui s'imposeront puissent être prises en compte, car il est important que cette CFTMEA soit le reflet de la pratique clinique des praticiens de terrain, comme elle l'a été jusque-là.

\section{Conflit d'intérêt : à compléter par l'auteur}

\section{Discussion}

D r Van Amerongen - Je vous suis reconnaissant de considérer les données de l'axe II. Pour les familles et l'entourage, la relation causaliste à une «maladie » survenue dans l'évolution du sujet malade risque d’entraîner un réductionnisme. 